\title{
SETS OF INTEGERS CONTAINING NO $n$ TERMS IN GEOMETRIC PROGRESSION
}

\author{
by J. RIDDELL†
}

(Received 3 June, 1968)

1. Introduction. R. A. Rankin [3] considered the problem of finding, for each integer $n \geqq 3$, a sequence of positive integers containing no $n$-term geometric progression. He constructed such sets $B_{n}$ having asymptotic density

$$
A_{n}=\frac{1}{\zeta(n-1)} \prod_{k=1}^{\infty} \frac{\zeta\left\{(2 n-3)^{k}\right\}}{\zeta\left\{(n-1)(2 n-3)^{k}\right\}} .
$$

For example $A_{3} \doteqdot 0.71975, A_{4} \doteqdot 0.8626$, and $A_{n} \rightarrow 1$ as $n \rightarrow \infty$.

Let $H(n)$ denote the class of all sequences of positive integers that contain no $n$-term geometric progression. Rankin wondered whether $A_{n}$ is the highest density possible for members of $H(n)$. In this paper we find members having higher density, in the cases $n \geqq 4$, and also find upper estimates for the possible density in all cases $n \geqq 3$.

If $E$ is a set of non-negative integers containing 0 , let $Q(E)$ denote the set of all integers $N$ of the form

$$
N=\prod_{i=1}^{\infty} p_{i}^{a_{i}}
$$

where $p_{i}$ is the $i$ th prime and each $a_{i}$ is chosen from $E$. We call $Q(E)$ the set of integers developed from the exponent choice set $E$. We shall simplify the notation by writing

$$
Q(\{a, b, \ldots\})=Q(a, b, \ldots) \text {. }
$$

If $E$ contains no $n$-term arithmetic progression, then $Q(E)$ contains no $n$-term geometric progression. Rankin's $B_{n}$ is the set $Q\left(C_{n}\right)$, where $C_{n}$ is the set of all non-negative integers which, when expressed in the scale of $2 n-3$, contain no digit greater than $n-2$.

For any real $x$ and set $Q$ of positive integers we let $Q(x)$ denote the number of elements of $Q$ that do not exceed $x$. If $Q$ has asymptotic density we shall denote it by $D(Q)$.

In Section 2, we either estimate or find the density of a member $Q\left(E_{n}\right)$ of $H(n)$ after proving the following lemma:

LEMMA 1. If $E$ is any exponent choice set, then $D(Q(E))$ exists.

For each $n \geqq 4$ we find a set $E_{n}$ such that $Q\left(E_{n}\right) \in H(n)$ and

$$
D\left(Q\left(E_{n}\right)\right)>A_{n}=D\left(Q\left(C_{n}\right)\right) \text {. }
$$

In fact for each $n \geqq 4$ we observe that there are many sets having these properties of $E_{n}$. In Section 3 we find an upper estimate for the possible density of members of $H(n)$ for each $n \geqq 3$. A table comparing some few of the densities we obtain with the corresponding upper estimates is included at the end of the paper.

† These results are contained in the author's Ph.D. thesis written at the University of Alberta in 1967 under the direction of Leo Moser. 
2. Members of $H(n)$ with density exceeding $A_{n}(n \geqq 4)$. We prove the following theorem.

THEOREM 1. (i) If $n$ is prime, there exists $Q \in H(n)$ such that

$$
D(Q)=\frac{\zeta(n)}{\zeta(n-1) \zeta\{(n-1) n\}} .
$$

(ii) If $n$ is composite, there exists $Q \in H(n)$ such that

$$
D(Q)>\frac{\zeta(n)}{\zeta(n-1) \zeta(h n)}-\left(\frac{1}{\zeta(h n-1)}-\frac{1}{\zeta(h n-h)}\right)
$$

where $h$ is the smallest prime divisor of $n$.

(iii) There exists $Q \in H(4)$ such that

$$
D(Q)>0.8952 \text {. }
$$

The estimate (3) is somewhat larger than that provided by (2) with $n=4$. We give the proof that the respective densities exceed $A_{n}$ in Section 2.1 . We first prove part (iii) of the theorem.

Proof of (iii). The set

$$
E_{4}^{\prime}: 0,1,2,4,5,7,8,9
$$

contains no 4-term arithmetic progression. We shall find a lower estimate for $D\left(Q\left(E_{4}^{\prime}\right)\right)$. The set $Q\left(E_{4}^{\prime}\right)$ will not contain $m$ if and only if there is a prime $p$ such that

$$
p^{3} \mid m \text { and } p^{4} \nmid m \text {, or } p^{6} \mid m \text { and } p^{7} \nmid m \text {, or } p^{10} \mid m \text {. }
$$

Given a prime $p$, the number of such $m$ not exceeding $x$ is

$$
K(x, p)=\left[\frac{x}{p^{3}}\right]-\left[\frac{x}{p^{4}}\right]+\left[\frac{x}{p^{6}}\right]-\left[\frac{x}{p^{7}}\right]+\left[\frac{x}{p^{10}}\right],
$$

and the density of the set of such numbers $m$ is

$$
K(p)=\lim _{x \rightarrow \infty} \frac{K(x, p)}{x}=\frac{1}{p^{3}}-\frac{1}{p^{4}}+\frac{1}{p^{6}}-\frac{1}{p^{7}}+\frac{1}{p^{10}} .
$$

By the principle of inclusion and exclusion,

$$
D\left(Q\left(E_{4}^{\prime}\right)\right)=1-\sum_{p} K(p)+\sum_{p<q} K(p) K(q)-\sum_{p<q<r} K(p) K(q) K(r)+\ldots,
$$

where the sums are respectively taken over all the tuples $(p),(p, q),(p, q, r), \ldots$ of primes satisfying the indicated inequalities. Since

$$
\begin{aligned}
& \sum_{p} K(p)<\sum_{p} \frac{1}{p^{3}}<1, \\
& \sum_{\substack{p<q<<<\ldots\} \\
(j \text { primes })}}(K(p) K(q) K(r) \ldots)<\sum_{p} K(p) \sum_{\substack{q<r<\ldots \ldots \\
(j-1 \text { primes })}}(K(q) K(r) \ldots)<\sum_{\substack{q<r<\ldots \ldots \\
(j-1 \text { primes })}}(K(q) K(r) \ldots),
\end{aligned}
$$


and

$$
\sum_{\substack{p<q<r<\ldots) \\(j \text { primes })}}(K(p) K(q) K(r) \ldots)<\left(\sum_{p} K(p)\right)^{j}=o(1) \text { as } j \rightarrow \infty .
$$

Hence the series (4) converges. We have estimated the first four terms and found that

$$
D\left(Q\left(E_{4}^{\prime}\right)\right)>1-0 \cdot 107569+0 \cdot 002875-0.000023>0.8952 .
$$

Before proceeding with the remaining parts of the theorem, we prove Lemma 1, using the method developed above.

Proof of Lemma 1. Given an exponent choice set $E$ and a prime $p$, we can define a quantity $K_{E}(p)$ corresponding to $K(p)$ above. If $E$ contains 1 , then the series $S$ in $K_{E}$ corresponding to (4) converges. For, the first term of $K_{E}(p)$ will be $1 / p^{a}$ with $a \geqq 2$, so that $\sum_{p} K_{E}(p)<\sum_{p} 1 / p^{2}<1$; hence we can obtain the inequalities (5) with $K_{E}$ in place of $K$. Therefore $D(Q(E))$ exists and is the sum of the series $S$. If $1 \notin E$, then $\sum_{p} K_{E}(p)$ diverges, for the first term of $K_{E}(p)$ is $1 / p$. However, in this case $D(Q(E))=0$, because $Q(E) \subset Q(0,2,3,4, \ldots)$, the set of squarefull numbers, and this set has density 0 . We refer the reader to the solution by $P$. T. Bateman [2] of a problem proposed by D. J. Newman which shows that, if $Q=Q(0,2,3,4, \ldots)$, then $Q(x)=O\left(x^{\frac{1}{2}}\right)$. Hence Lemma 1 .

Proof of (i). If $\boldsymbol{n}$ is prime, then the set

$$
\begin{aligned}
& 0, \quad 2, \ldots, \quad n-2 \text {, } \\
& n, \quad n+1, \quad n+2, \ldots, \quad 2 n-2 \text {, } \\
& E_{n}: \quad 2 n, \quad 2 n+1, \quad 2 n+2, \ldots, \quad 3 n-2 \text {, } \\
& (n-2) n, \quad(n-2) n+1, \quad(n-2) n+2, \ldots,(n-1) n-2
\end{aligned}
$$

contains no $n$-term arithmetic progression. For if $E_{n}$ contained such progressions, one of them would have its first term among $0,1, \ldots, n-2$, and all of them would have common difference less than $n$. However, if $0 \leqq a \leqq n-2$ and $1 \leqq d \leqq n-1$, some term of the progression

$$
a, \quad a+d, \quad a+2 d, \ldots, a+(n-1) d
$$

is congruent to -1 modulo $n$ and hence is not in $E_{n}$. This is because $(d, n)=1$, whence $0, d, 2 d, \ldots,(n-1) d$ form a complete residue system modulo $n$.

Now, with $s=\sigma+i t(\sigma, t$ real $)$,

$\sum_{N \in Q\left(E_{n}\right)} \frac{1}{N^{s}}=\prod_{p}\left(\sum_{a \in E_{n}} \frac{1}{p^{a s}}\right)=\prod_{p} \frac{1-1 / p^{(n-1) s}}{1-1 / p^{s}} \frac{1-1 / p^{(n-1) n s}}{1-1 / p^{n s}}=\frac{\zeta(s) \zeta(n s)}{\zeta\{(n-1) s\} \zeta\{(n-1) n s\}}$. 
We now employ the following lemma (see Ayoub [1]):

LEMMA 2. If

$$
f(s)=\sum_{N=1}^{\infty} \frac{a(N)}{N^{s}} \quad \text { and } \quad \lim _{x \rightarrow \infty} \frac{\sum_{N \leqq x} a(N)}{x}=A \text {, }
$$

then

$$
\lim _{s \rightarrow 1}(s-1) f(s)=A
$$

Defining

$$
a(N)=\left\{\begin{array}{l}
1 \text { if } N \in Q\left(E_{n}\right) \\
0 \text { otherwise }
\end{array}\right.
$$

we have

$$
\sum_{N \in Q\left(E_{n}\right)} \frac{1}{N^{s}}=\sum_{N=1}^{\infty} \frac{a(N)}{N^{s}},
$$

and with $Q=Q\left(E_{n}\right)$,

$$
Q(x)=\sum_{N \leqq x} a(N) .
$$

Lemma 1 assures us that $D\left(Q\left(E_{n}\right)\right)=\lim _{x \rightarrow \infty} Q(x) / x$ exists, and by Lemma 2 we can find this limit from (7). It is the residue of (6) at the simple pole $s=1$. Thus

$$
D\left(Q\left(E_{n}\right)\right)=\frac{\zeta(n)}{\zeta(n-1) \zeta\{(n-1) n\}},
$$

and hence part (i) of Theorem 1.

Note that we could adjoin integers to the above set $E_{n}$ and still have a set free of $n$-term progressions, thus obtaining an even denser member of $H(n)$.

Proof of (ii). Suppose that $n$ is composite, and that $h$ is the smallest prime divisor of $n$. Then the set

$$
\begin{aligned}
& 0, \quad 1, \quad 2, \ldots, \quad n-2 \text {, } \\
& n, \quad n+1, \quad n+2, \ldots, \quad 2 n-2 \text {, } \\
& E_{n} \text { : } \\
& (h-2) n, \quad(h-2) n+1, \quad(h-2) n+2, \ldots,(h-1) n-2 \text {, } \\
& (h-1) n, \cdot(h-1) n+1, \quad(h-1) n+2, \ldots, h n-h-1
\end{aligned}
$$

contains no $n$-term arithmetic progression. For consider any progression with first term $a$ and common difference $d$ such that

$$
0 \leqq a<a+d<a+2 d<\ldots<a+(n-1) d \leqq h(n-1)-1 .
$$


Evidently $d<h$. Hence $(d, n)=1$. Therefore $a, a+d, \ldots, a+(n-1) d$ form a complete residue system modulo $n$, whence this progression contains one of $n-1,2 n-1, \ldots,(h-1) n-1$, and is not contained in $E_{n}$.

We shall obtain the lower estimate (2) for $D\left(Q\left(E_{n}\right)\right)$. The numbers $h n-h, h n-h+1, \ldots$, $h n-2$ cannot be included in $E_{n}$ since each of these is the $n$th term of an arithmetic progression having difference $h$ and first $n-1$ terms in $E_{n}$. Using Lemma 2 we find that

$$
D\left(Q\left(E_{n} \cup\{h n-h, h n-h+1, \ldots, h n-2\}\right)\right)=\frac{\zeta(n)}{\zeta(n-1) \zeta(h n)} .
$$

and (2) will follow when we make allowance for the exclusion of $h n-1, h n-h+1, \ldots, h n-2$.

Given any exponent choice set $E$ and set of positive integers $F$ disjoint from $E$, we shall denote by $Q(E \& F)$ the set of integers developed from the exponent choice set $E \cup F$ with always at least one element from each of $E$ and $F$ included among the exponents chosen (we always include the 0 from $E$ ). Then

$$
Q(E) \cup Q(E \& F)=Q(E \cup F) .
$$

By Lemma $1, D(Q(E))$ and $D(Q(E \cup F))$ exist. Therefore, since the sets on the left side of (9) are disjoint, $D(Q(E \& F)$ exists and

$$
D(Q(E))+D(Q(E \& F))=D(Q(E \cup F)) .
$$

Now, writing $G=\{h n-h, h n-h+1, \ldots, h n-2\}$, we have by (10)

$$
D\left(Q\left(E_{n}\right)\right)=D\left(Q\left(E_{n} \cup G\right)\right)-D\left(Q\left(E_{n} \& G\right)\right) \text {. }
$$

Furthermore,

$$
Q\left(E_{n} \& G\right) \subset Q(\{0,1,2, \ldots, h n-h-1\} \& G)=Q(0,1,2, \ldots, h n-2)-Q(0,1,2, \ldots, h n-h-1),
$$

where we have applied (9). Hence, by (10),

$$
\begin{aligned}
D\left(Q\left(E_{n} \& G\right)\right) & \leqq D(Q(0,1,2, \ldots, h n-2))-D(Q(0,1,2, \ldots, h n-h-1)) \\
& =\frac{1}{\zeta(h n-1)}-\frac{1}{\zeta(h n-h)} .
\end{aligned}
$$

Hence, from (8) and (11), the result follows, and the proof of Theorem 1 is now complete.

One can again adjoin integers to $E_{n}$, in the case $n$ is composite, and obtain a still denser member of $H(n)$. For example if $n$ is even, and $l$ is the smallest prime divisor of $n-1$, then the set

$$
E_{n}^{\prime}=\{0,1,2, \ldots, l(n-1)\}-\{n-1,2(n-1), 3(n-1), \ldots,(l-1)(n-1)\}
$$

contains no $n$-term arithmetic progression. We found earlier that $D\left(Q\left(E_{4}^{\prime}\right)\right)>0.8952$. By comparison the estimate (2) in the case $n=4$, found using $E_{4}=\{0,1,2,4,5\}$, is 0.88796 to five places, and estimating from above, we find using (10) that

$$
D\left(Q\left(E_{4}\right)\right)<1 / \zeta(6)-[1 / \zeta(4)-1 / \zeta(3)]<0.89093 .
$$




\section{J. RIDDELL}

2.1. Comparison of the densities. We shall show that

$$
\frac{\zeta(n)}{\zeta(n-1) \zeta(2 n)}-\left[\frac{1}{\zeta(2 n-1)}-\frac{1}{\zeta(2 n-2)}\right]>A_{n}
$$

for $n \geqq 4$, and that

$$
\frac{\zeta(n)}{\zeta(n-1) \zeta(h n)}-\left[\frac{1}{\zeta(h n-1)}-\frac{1}{\zeta(h n-h)}\right]>\frac{\zeta(n)}{\zeta(n-1) \zeta\{(h-1) n\}}
$$

for $3 \leqq h \leqq n-2$. From (12) it will follow that the density in Theorem 1(ii), in the case $n$ is even, exceeds $A_{n}$. If $n$ is an odd composite number and $h$ is the smallest prime divisor of $n$, then $3 \leqq h \leqq \sqrt{ } n$ and (13) will hold. Furthermore in this case the right side of (13), and hence that of $(2)$, exceeds $\zeta(n) /(\zeta(n-1) \zeta(2 n))$, as does the quantity $\zeta(n) /[\zeta(n-1) \zeta\{(n-1) n\}]$ of $(1)$ in the case $n$ is prime. Hence by (12) the densities in Theorem 1(i), (ii) will have been shown to exceed $A_{n}$ in any case.

We use the following easily proved lemma:

LEMMA 3. For integers $a>1$ and $b>0$,

$$
\zeta(a+b)<1+\frac{\zeta(a)-1}{2^{b}} .
$$

To prove (12) we first show that

$$
\prod_{k=1}^{\infty} \frac{\zeta\left\{(2 n-3)^{k}\right\}}{\zeta\left\{(n-1)(2 n-3)^{k}\right\}}<\frac{\zeta(2 n-4)}{\zeta\left(2 n^{2}\right)} \text { for } n \geqq 3
$$

and then that

$$
\frac{2 n-4}{\zeta\left(2 n^{2}\right)}<\frac{\zeta(n)}{\zeta(2 n)}-\zeta(n-1)\left[\frac{1}{\zeta(2 n-1)}-\frac{1}{\zeta(2 n-2)}\right]
$$

for $n \geqq 5$. This will imply (12) for $n \geqq 5$. For $n=4$ we find, using tables, that the left side of (12) exceeds 0.88796 while $A_{4}<0.8627$.

We observe that

$$
\zeta\left(2 n^{2}\right)<\zeta\{(n-1)(2 n-3)\}<\prod_{k=1}^{\infty} \zeta\left\{(n-1)(2 n-3)^{k}\right\},
$$

so that if we prove

$$
\prod_{k=1}^{\infty} \zeta\left\{(2 n-3)^{k}\right\}<\zeta(2 n-4)
$$

for $n \geqq 3$ we shall have (14). Writing $m=2 n-3$, we shall prove

$$
\zeta(m-1)>1+2(\zeta(m)-1)>\prod_{k=1}^{\infty} \zeta\left(m^{k}\right)
$$

for $m \geqq 3$, and this will yield (16). The first inequality of (17) is immediate from Lemma 3 . 
Again, from the lemma,

$$
\prod_{k=1}^{\infty} \zeta\left(m^{k}\right)<\prod_{k=1}^{\infty}\left(1+\frac{\zeta(m)-1}{2^{m^{k}-m}}\right),
$$

and writing $x=2^{m}(\zeta(m)-1)$ one can show by comparing logarithms that

$$
\prod_{k=1}^{\infty}\left(1+\frac{x}{2^{m^{k}}}\right)<1+\frac{x}{2^{m-1}}
$$

for $m \geqq 3$, whence follows the second inequality of (17).

The inequality (15) is equivalent to

$$
\frac{\zeta(2 n-4)}{\zeta\left(2 n^{2}\right)}+\zeta(n-1) \frac{\zeta(2 n-2)-\zeta(2 n-1)}{\zeta(2 n-1) \zeta(2 n-2)}<\frac{\zeta(n)}{\zeta(2 n)},
$$

and the second term on the left side here is less than $\zeta(n-1)(\zeta(2 n-2)-1) / \zeta^{2}(2 n)$. Replacing that second term by this quantity and multiplying through by $\zeta^{2}(2 n) \zeta\left(2 n^{2}\right)$, we find that the left side of the resulting inequality is less than

$$
\zeta(2 n) \zeta^{2}(2 n-4)+\zeta(2 n) \zeta(n-1)(\zeta(2 n-2)-1) .
$$

For $n \geqq 6$ one can show by Lemma 3 that this quantity is less than $\zeta(n) \zeta(2 n) \zeta\left(2 n^{2}\right)$, giving (18) for $n \geqq 6$, while for $n=5$, (18) can be proved using tables. Hence (12).

The proof of (13) involves manipulations similar to those in the proof of (15).

3. Upper estimates. Let

$$
M_{n}=\sup \left\{\lim _{x \rightarrow \infty} \frac{Q(x)}{x} \mid Q \in H_{1}(n)\right\},
$$

where $H_{1}(n)$ is that subset of $H(n)$ whose members have asymptotic density. We prove the following theorem.

THEOREM 2. For every $n \geqq 3$,

$$
M_{n} \leqq 1-\frac{1}{2^{n}-1} \text {. }
$$

The proof of Theorem 2 depends on Theorem 3 below, which is concerned with geometric progressions of integral ratio $r$. Let $I$ denote the set of positive integers. For any integer $r>1$ let $R=R(n, r)$ denote the set of geometric progressions in $I$ of $n$ terms and ratio $r$, and let $H(n, r)$ denote the class of all sequences in $I$ that contain no progression of $R$. Further, let $H_{1}(n, r)$ be the class of all sequences $Q \in H(n, r)$ for which $\lim Q(x) / x$ exists. We define

$$
\begin{aligned}
& M_{n, r}^{*}=\sup \left\{\limsup _{x \rightarrow \infty} \frac{Q(x)}{x} \mid Q \in H(n, r)\right\}, \\
& M_{n, r}=\sup \left\{\lim _{x \rightarrow \infty} \frac{Q(x)}{x} \mid Q \in H_{1}(n, r)\right\} .
\end{aligned}
$$


THEOREM 3. (i) No integer appears in more than $n$ progressions of $R$.

(ii) There is exactly one subset of I with the property: Each element of the set appears in $n$ progressions of $R$ and each progression of $R$ contains exactly one element of the set.

(iii) Let $S$ be the set in (ii). If $T \subset I$ and $I-T \in H(n, r)$, then $T(x) \geqq S(x)$ for every $x$.

(iv) $I-S \in H_{1}(n, r)$ and $\lim S(x) / x=(r-1) /\left(r^{n}-1\right)$.

(v) $M_{n, r}=M_{n, r}^{*}=1-(r-1) /\left(r^{n}-1\right)$.

If analogously to $M_{n, r}^{*}$ we defined

$$
M_{n}^{*}=\sup \left\{\limsup _{x \rightarrow \infty} \frac{Q(x)}{x} \mid Q \in H(n)\right\},
$$

we might expect that similarly $M_{n}=M_{n}^{*}$. Perhaps this would be so if one considered only geometric progressions with integral ratio, but it seems doubtful in the general case.

Proof of Theorem 3. Let us separate $R$ into families $F_{k}$ of progressions:

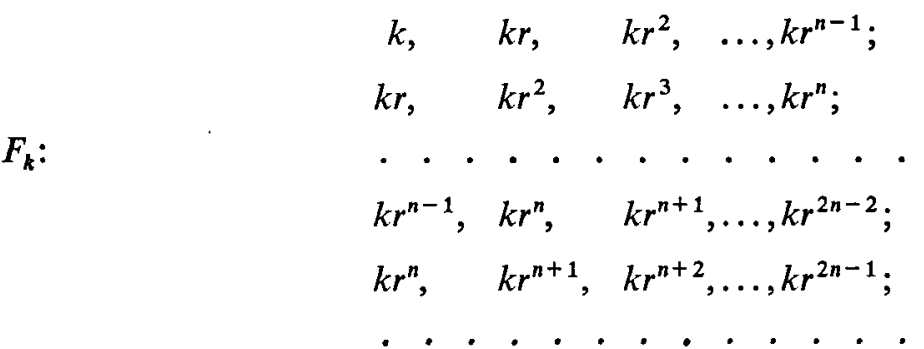

where $k \in I, r \nmid k$. Clearly $\bigcup_{\substack{k=1 \\ r \nmid k}}^{\infty} F_{k}=R$, and no integer appears in more than $n$ progressions of one family. Furthermore, if $V_{k}$ denotes the set of all integers appearing in the progressions of $F_{k}$, then the sets $V_{k}$ are pairwise disjoint. For if $k r^{u}=l r^{v}$ and $k \neq l$, then $u \neq v$ and either $r \mid k$ or $r \mid l$. (i) follows.

The integers $k r^{n-1}, k r^{2 n-1}, k r^{3 n-1}, \ldots$ each appear in exactly $n$ of the progressions of $F_{k}$, and each progression of $F_{k}$ contains exactly one of them; it is clear that this is the only set of integers with this property. Since the $V_{k}$ are pairwise disjoint, the set

$$
S=\bigcup_{\substack{k=1 \\ r \nmid k}}^{\infty}\left\{k r^{n-1}, k r^{2 n-1}, k r^{3 n-1}, \ldots\right\}
$$

has the property required in (ii). It is clear that $I-S \in H(n, r)$.

Proceeding to (iii), we observe that if each $F_{k}$ is separated into blocks of $n$ progressions each, starting with the first member of the family, then in order that $I-T \in H(n, r), T$ must contain at least one integer from each block of each family. Since $S$ contains exactly one integer from each block, $T(x) \geqq S(x)$ for every $x$. 
The number of integers

$$
r^{i n-1}, 2 r^{i n-1}, \ldots,(r-1) r^{i n-1},(r+1) r^{i n-1}, \ldots
$$

not exceeding $x$ is

$$
\begin{aligned}
a_{i} & =\left[\frac{x-r^{i n-1}}{r^{i n}}+1\right]+\left[\frac{x-2 r^{i n-1}}{r^{i n}}+1\right]+\ldots+\left[\frac{x-(r-1) r^{i n-1}}{r^{i n}}+1\right] \\
& =\left[\frac{x}{r^{i n}}+\frac{r-1}{r}\right]+\left[\frac{x}{r^{i n}}+\frac{r-2}{r}\right]+\ldots+\left[\frac{x}{r^{i n}}+\frac{1}{r}\right]
\end{aligned}
$$

provided that $1 \leqq i \leqq m=\left[\frac{\log _{r} x+1}{n}\right]$, while if $i>m$, the integers (19) all exceed $x$. Hence

$$
S(x)=\sum_{i=1}^{m} a_{i}=\sum_{i=1}^{m} \frac{(r-1) x}{r^{i n}}+O(\log x),
$$

so that $S$ has density

$$
\lim _{x \rightarrow \infty} \frac{S(x)}{x}=\sum_{i=1}^{\infty} \frac{r-1}{r^{i n}}=\frac{r-1}{r^{n}-1}
$$

and we have proved (iv).

From (iv), $M_{n, r} \geqq 1-(r-1) /\left(r^{n}-1\right)$. On the other hand, by (iii), if $U \in H(n, r)$, then $U(x) \leqq[I-S](x)$ for every $x$, so that $M_{n, r}^{*} \leqq 1-(r-1) /\left(r^{n}-1\right)$. Since $M_{n, r} \leqq M_{n, r}^{*}$ by definition, (v) follows.

Proof of Theorem 2. The theorem follows immediately from the observation that $M_{n} \leqq M_{n, r}$ for any $r$. We choose $r=2$ since $M_{n, r}$ is smallest for that value of $r$.

In the case $n=3$ we have obtained the better estimate

$$
M_{3}<0.8339 \text {. }
$$

This compares with the estimate $6 / 7=0 \cdot 8571 \ldots$ of Theorem 2 . We find (20) by considering what integers must be removed from $I$ in order to eliminate, in addition to all 3-term progressions of ratio 2, certain progressions of ratio 3. The details are too lengthy to be included here.

The most dense members of $H(n)$ discussed in Sections 1 and 2 provide lower estimates for $M_{n}$. We compare these with our upper esimates for $M_{n}$ for some few values of $n$ :

$\begin{array}{ccc}n & \text { lower estimate } & \text { upper estimate } \\ 3 & A_{3}=0.7197 \ldots & 0.8339 \\ 4 & 0.8952 & 14 / 15=0.933 \dot{3} \\ 5 & 0.9580 & 30 / 31=0.9677 \ldots \\ 8 & 0.9957 & 254 / 255=0.9960 \ldots\end{array}$




\section{REFERENCES}

1. R. Ayoub, An introduction to the analytic theory of numbers, Mathematical Surveys, No. 10 . American Mathematical Society (Providence, 1963), p. 86.

2. P. T. Bateman, Solution to Problem 4459 [1951, p. 636], American Math. Monthly 61 (1954), 477-479.

3. R. A. Rankin, Sets of integers containing not more than a given number of terms in arithmetical progression, Proc. Roy. Soc. Edinburgh Sect. A 65 (1962), 332-344.

UNIVERSITY OF VICTORIA

VICTORIA, B.C., CANADA 